(C) Springer-Verlag 2009

Winfried A. Willinek

\title{
State-of-the-art MRI with Blood Pool Agents: New perspectives for advanced vascular imaging
}

\section{Preface}

For many years, digital subtraction angiography was the procedure of choice in the diagnostic workup of vascular disease, mainly because a safe, non-invasive, operatorindependent alternative was not available. One of the notable developments in non-invasive imaging of the vascular system has been magnetic resonance angiography (MRA). Although promising, limitations of noncontrast-enhanced techniques precluded its widespread acceptance in clinical practice. In recent years contrastenhanced MRA (CE-MRA) has helped to overcome many limitations, and MRA using extracellular contrast media (ECCM) has become a first-line tool for vascular imaging. Nevertheless, DSA still is considered the standard of reference due to its superior spatial resolution. Spatial resolution was limited in standard CE-MRA with extracellular contrast agents because of the intrinsic limitation of the acquisition window that has to correspond with the arterial bolus passage ("first-pass imaging").

With the advent of an intravascular ("blood pool") contrast agent, very high spatial resolution can now be obtained in CE-MRA because of the possibility of dramatically increasing the acquisition time. Gadofosveset (Vasovist ${ }^{\circledR}$, Bayer Schering Pharma AG, Berlin /Germany) is the first intravascular contrast agent which has been approved for use with magnetic resonance angiography in the European Union, Turkey, Switzerland, Australia and Canada; it is approved for aortoiliac MRA in the USA since December 2008. Gadofosveset reversibly binds to albumin, providing extended intravascular enhancement compared with existing extracellular magnetic resonance contrast agents. The albumin binding prolongs the imaging time for the contrast agent in the steady state up to more than 1 hour and provides the highest relaxivity of currently available contrast agents. The prolonged available acquisition time can be exploited to acquire

Winfried A. Willinek, Department of Radiology, University of Bonn, Sigmund-Freud-Strasse 25, 53105 Bonn, Germany, Tel.: +49-228-287-15960, Fax: +49-228-287-11831,

e-Mail:winfried.willinek@ukb.uni-bonn.de additional high-resolution images during the steady state, yielding isotropic sub-millimetre resolution. Furthermore, multiple vascular beds, including the venous system, can theoretically be imaged with very high spatial resolution after one single injection of the blood pool contrast agent as compared with two or more injections that would be needed with extracellular contrast agents.

After being injected, these extracellular agents rapidly diffuse out of the vasculature into the interstitial space. Their initial distribution half-life is only about 15 minutes, which leads to the following practical constraints:

- As the window for imaging is limited to 1-3 minutes, the CE-MRA acquisition needs to be performed during peak arterial enhancement, which makes timing of the MRA sequence crucial to avoid edge enhancement artefacts or venous contamination that occur if the data collection is started either too early or too late.

- Vessel-to-background contrast may be limited because of the relatively low relaxivity rate of standard extracellular contrast agents. To compensate for this, double or even triple doses of these agents have been used in clinical practice.

Because of its high relaxivity, gadofosveset is injected at a recommended very low dosage of $0.03 \mathrm{mmol} / \mathrm{kg}(0.12 \mathrm{ml} /$ $\mathrm{kg}$ ) body weight, yielding a single dose between 5 and $15 \mathrm{ml}$ in the majority of patients while still producing excellent blood-versus-tissue contrast enhancement.

As opposed to imaging during initial arterial passage of the contrast agent, MRA with a blood pool contrast agent in the equilibrium phase or steady state leads to the enhancement not only of arteries, but also of veins. This might seem to be cumbersome. However, the spatial resolution of steady-state images is typically very high, which ensures that separation of arteries and veins is actually quite easy when source images are used as multiple reformats instead of maximum intensity projections (MIP). Furthermore, the simultaneous depiction of veins can actually assist pre-operative planning by providing vascular surgeons with information about the suitability of autologous veins for use as bypass grafts. 
In summary, MRA with blood pool contrast agents opens new horizons for non-invasive vascular imaging. This special issue is dedicated to state-of-the-art magnetic resonance imaging using the blood pool contrast agent gadofosveset. It includes new perspectives of advanced vascular imaging such as comprehensive imaging of deep venous thrombosis and pulmonary embolism, imaging of the diabetic foot, supra-aortic, neuro and plaque imaging and peripheral MRA with improved diagnostic accuracy.

I would like to thank the various experts in the field for their contributions, and I hope that the esteemed reader will be stimulated by the versatile clinical applications and imaging protocols to integrate the new concept of vascular imaging with blood pool agents in his/her own clinical practice.

Winfried A. Willinek 